\title{
Virtual Reality Program for Enhancing Learning in a High School Human Biology Course
}

\author{
José Garza-Martínez* \\ Tecnologico de Monterrey, Escuela Nacional de Preparatoria, Mexico
}

\begin{abstract}
The creation of virtual environments and their implementation have been a matter of greatest importance in the last decades, becoming a breaking point for progress in different fields like communication, entertainment, and education. Additionally, the learning and teaching of human biology have been more relevant through the increase of biology-related research and medical advances. However, learning human biology, especially anatomy, is perceived as difficult by students. The objective of this study is to improve the learning of human biology by implementing a Virtual Reality Program (VRP) in a high school human biology course, measuring academic performance in a final knowledge evaluation. The VRP used immersive Virtual Reality (VR) devices (Oculus-Go® and Oculus-Rift ${ }^{\circledR}$ ) and consisted of three stages: exploration, virtual reality lab, and knowledge reinforcement. In the exploration, the students had a guided approach of the topics with a professor's explanation; afterward, they had guided and independent collaborative experience in the Virtual Reality lab; and finally, their knowledge was reinforced in communication with the professor. A post-test only with a control group design was employed with 120 students. There were two experimental groups (63 students) and two control groups (57 students). After completing the stages of the VRP, all the groups had a final knowledge evaluation where the experimental groups obtained a mean of 77.24, while the control groups had 69.11. There was a significant statistical difference between the groups with a $\mathrm{P}<0.05$ using a two-sample t-test. This proves that implementing a long-time and collaborative immersive VR approach in high school human biology courses is effective for enhancing learning and improving academic performance.
\end{abstract}

Keywords: educational innovation, virtual reality, active learning, academic performance

\section{Introduction}

Nowadays, education is being revolutionized by technology-enhanced learning platforms. Knowledge must become life standing, that's why learning goals should consider the learning taxonomy for students to understand, remember, and apply knowledge (Fink, 2013). Academic performance is defined as a measure of how well students have completed an assessment set, this concept is a complex phenomenon influenced by multiple factors like meta reflective thinking and learning, motivation, study skills versus disengagement, quality of instruction, and socioeconomic status (Mason, 2017).

Jaron Lanier was the first person to define VR as a technology that creates three-dimensional spaces employing a computer, thus allowing the simulation of reality with the advantage of perfecting the desired objective (Martínez, 2011). Currently, the definitions for VR are numerous, perhaps for being in the popular press like the television or any other gadget (Arbona et al., 2007). For producing virtual experiences, VR software with the content of the virtual world and a VR device to visualize and move in the virtual world are needed; this medium provides an important tool that can help in education, science, and many other fields (Steuer, 1992). There are three main categories of VR environments: MUD (Multiuser Dungeon); MOO (Multi-user domain, ObjectOriented), and Immersive VR. MOO focus on environments for online activities and graphical reality; Immersive VR uses virtual representations and symbolizes presence in virtual reality. All categories have applications for educational purposes (Solak \& Erdem, 2015). Meaningful learning, especially using VR, requires qualities like flexibility, creativity, and responsibility to provide a conducive learning environment. 
Joe Blatt expressed the importance of children learning as soon as possible that what is on the other side of the screen (Martínez, 2011). The education of future generations in a computerized society is a mission that must be assumed with responsibility. Solid and flexible education must adapt to changing conditions; VR technology has grown in popularity as a technological advance that can support the teaching process since it allows students to observe events at different scales, visualize concepts abstracts or any condition that is not possible to carry out in real conditions (Escartín, 2000). In countries with a high educational development index, multiple programs have been established to introduce many students and educators to VR technology (Youngblut, 1998). In addition, academic institutions have developed programs for VR research, finding advantages in skills like observation, laboratory thinking, a higher response of students, instant access to information, and integration of multiple concepts for motivating students (Shudayfat, et al. 2013).

Institutions must incorporate technologies in education for enhancing learning. Some programs guide teachers and students regarding the use of VR technology, like Virtual Education Science and Math of Texas (VESAMOTEX) (Youngblut, 1998). Students need to have real practice conditions and gain skills related to their professional fields during their education, VR programs can help at being applied on relevant practical topics like animal testing (Arslan et al., 2020).

The applications of VR in biology learning biology are unlimited. A desktop VR-based learning environment was created for examining the effectiveness of adding a generative learning strategy with a VR lesson for improving spatial abilities (Lee \& Wong, 2014). A biology class with VR simulations of multiple organs of the human body was proposed, finding that students with the traditional learning method have more difficulties in learning (Shudayfat, et al. 2013). Three-dimensional VR technology learning programs were designed for middle school students finding that simulations increase their interest and understanding of scientific concepts (Shim et al., 2003). A guide to creating VR environments in education using multiple scenarios was published (García et al., 2019).

The reported VR projects focused mainly on comparing the students' appreciation of the VR experience against a control group, as a measurement of the implementation's efficacy; Shim et al. (2003) reported the impact on students' interest and Bennet \& Saunders (2019) used an experience appreciation survey. On the other hand, there are reported results using the participants' perceptions as a tool for exploring learners' methodology aiming at developing VR experiences (García et al., 2019) (Youngblut, 1998) or to determine the learning effectiveness of skills (Lee \& Wong, 2014). A project focused on increasing academic performance in a standalone manner comparing the results of a single session implementation with traditional methods (Shudayfat, et al., 2013).

The previous projects used additional multimedia material to traditional courses in a short-time implementation with an individual or pairs working format. There are several projects of VR implementation; however, there is no reported information of long-time implementations focusing on students' academic performance, nor reported publications including collaborative activities using VR approaches.

This research focused on using a long-time VR implementation with collaboration elements within students for enhancing learning by improving academic performance.

\section{Research objective and hypothesis}

The hypothesis from this research is that long-time and collaborative immersive VR implementations could be effective for improving learning on a high school human biology course. 
The objective of the research is to improve the learning of human systems and their functions by implementing an immersive VR Program of 10 sessions during a semester (17 weeks) on a high school human biology course, leading to an improvement of the students' academic performance in a final knowledge evaluation

\section{Methodology}

\section{Research design}

The research was made with a quantitative approach, focusing on the measurement of students' academic performance. The target population was 120 first-year students from high school level in Tecnologico de Monterrey, Eugenio Garza Lagüera Campus in the city of Monterrey, Mexico. The students were from a single high school with ages between 15 to 17 years. The research was conducted throughout an academic semester of 17 weeks with 47 sessions of 50 minutes. A 10 sessions implementation was performed between week 5 and week 15 .

A post-test only with a control group design was employed. There were two experimental groups (63 students) and two control groups (57 students). Only the experimental groups participated in the implementation. After completing the semester, all groups had a final knowledge evaluation from the human biology course to measure their academic performance and evaluate the efficacy of the implementation.

\section{Implementation}

\section{Structure}

The proposal consisted in creating a Virtual Reality Program (VRP) complementary to the academic knowledge content of a high school human biology course; which is divided into four blocks and three evaluation weeks, it is focused on the learning of concepts related to human biology, including understanding anatomy and diseases (Vicerrectoria academica ITESM, 2014). The VRP had the objective of enhancing the significant learning of concepts seen during all the semester (Figure 1). The students participated in the VR sessions and completed collaborative activities using the technology.

\section{Time(Weeks)}

\section{\begin{tabular}{|l|l|l|l|l|l|l|l|l|l|l|l|l|l|l|l|l|}
\hline W1 & W2 & W3 & W4 & W5 & W6 & W7 & W8 & W9 & W10 & W11 & W12 & W13 & W14 & W15 & W16 & W17
\end{tabular}}

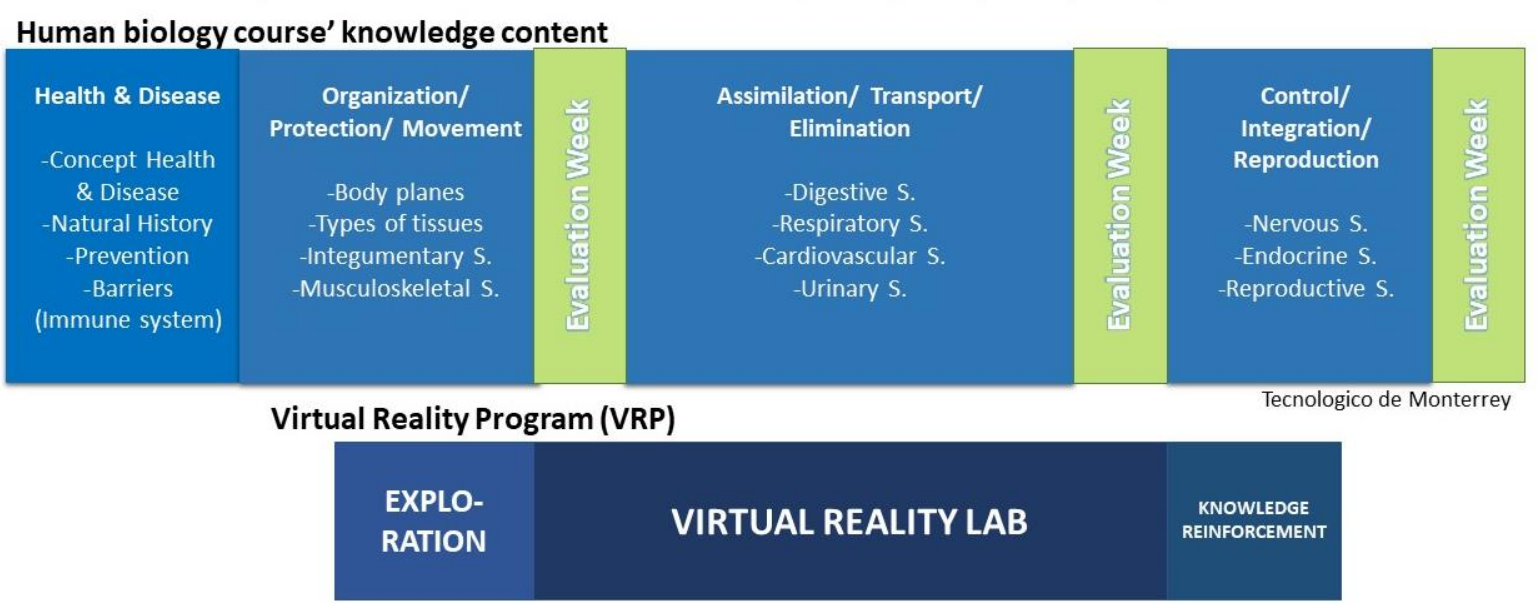

Figure 1. Virtual Reality Program's implementation in the context of the knowledge content in human biology's course 
The VRP was divided into three stages, each had a specific objective:

- Exploration. Introducing and guiding students to the VR environment.

- Virtual Reality Lab (VR Lab). Allowing students to work collaboratively using VR environments.

- Knowledge reinforcement. Sharing experiences in VR environments and reviewing learned concepts.

Each stage was organized in a different amount of sessions, where the activities could vary in approach (guided, partially guided, or self-guided), objective (demonstration, recognition or concept's review), and materials (VR device and VR software) (Figure 2).
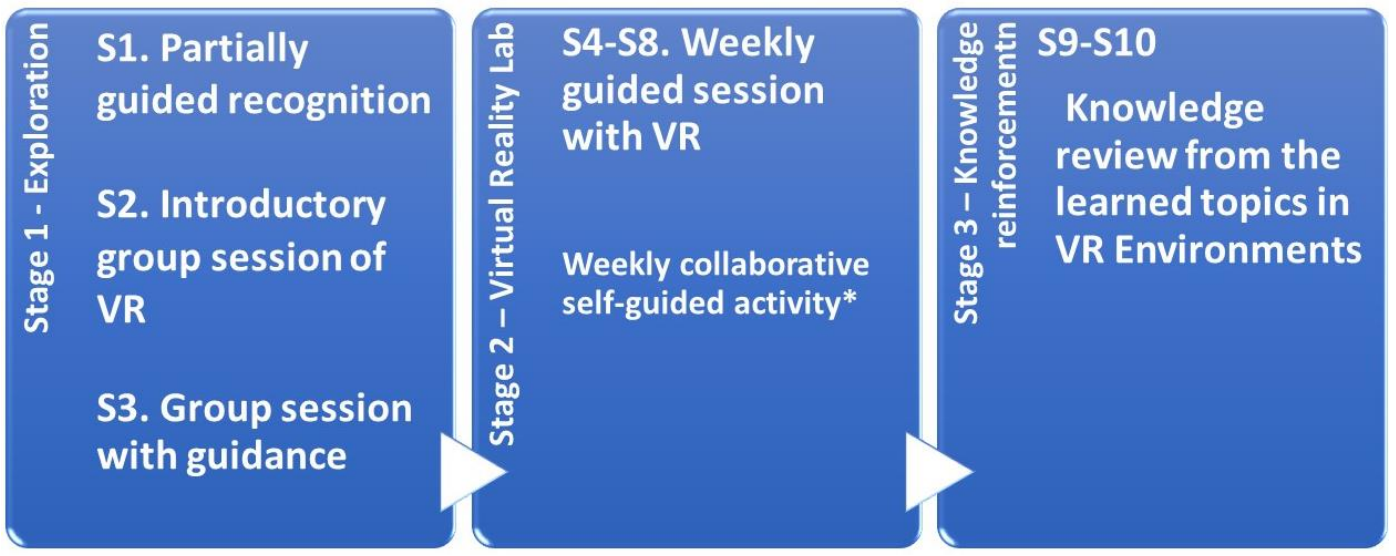

Figure 2. Lesson plan of Virtual Reality Program divided into stages and amount of sessions.

\section{Stage 1. Exploration}

In this stage, the students were introduced during three sessions to the VR environment with a guided, partially guided, and self-guided approach.

In the first session, the students had a partially guided approach, intending to interact with the VR environment, recognize tools and features of the software. The objective was recognition, working with the topic "body planes”. For materials, the students used the VR device Oculus-Go®, which consists of googles for observing the interphase and hand controls for moving and selecting, with the VR software Human Anatomy VRß, where the parts of the human body can be observed and selected (Figure 3).

The second session used a guided approach, introducing the students to the materials for the rest of the VRP: the VR device Oculus-Rift ${ }^{\circledR}$, where the users have an enhanced immersion with the googles and a better interaction with two hand controllers, and the VR software Organon 3D VR Anatomy®, where multiple human systems can be selected and separate their components for a better appreciation (Figure 3). The objective was a demonstration, explaining with VR the topic "musculoskeletal system" by analyzing and identifying the main organs of those systems.

The third session aimed to make the students interact with the software and demonstrate its usage. A demonstrative objective with the same materials was used having a partially guided approach. Three students were asked to identify the parts and organs of the "musculoskeletal system". 


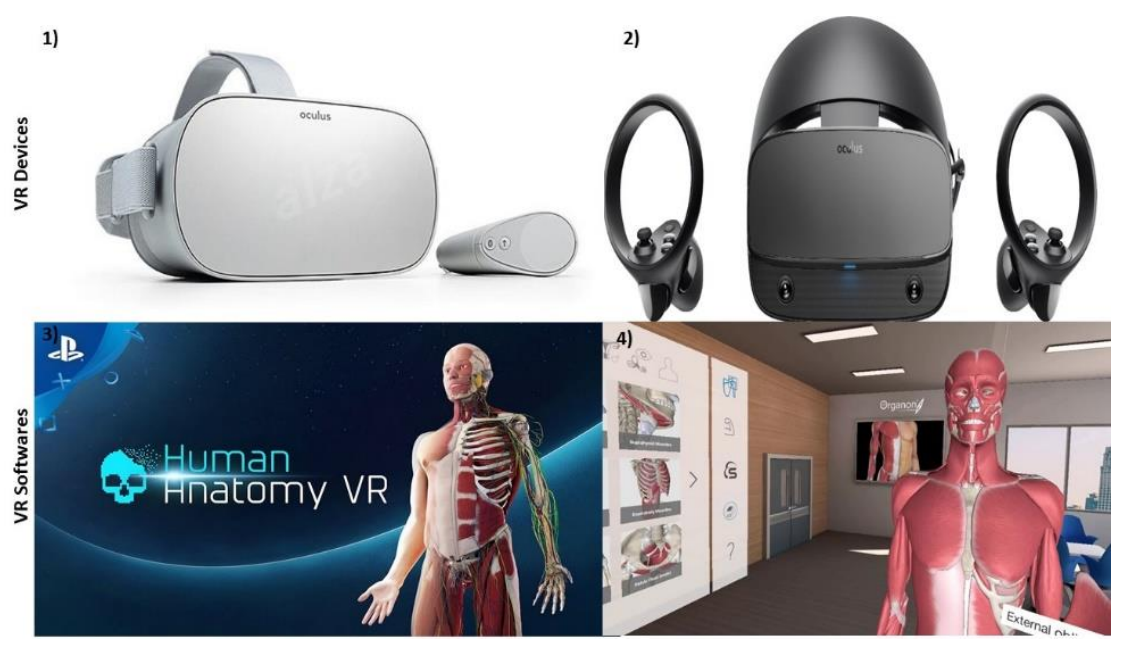

Figure 3. VR devices and VR software used in the implementation. 1) Oculus-Go® - Standalone Virtual Reality Headset by Oculus from Facebook (2020). 2) Oculus-Rift ${ }^{-}$- Virtual Reality Headset by Oculus from Facebook (2020). 3) Human Anatomy VR® by Playstation (2020). 4) Organon 3D VR Anatomy® by Medis Media (2020).

\section{Stage 2. Virtual Reality Lab}

In the second stage, the Virtual Reality Lab had two approaches: weekly guided sessions and collaborative selfguided activities. This stage took place during session 4 and session 8 of the VRP.

The course content related to the stage was: digestive system, respiratory system, cardiovascular system, and urinary systems. Each content was learned weekly in three sessions; the first and second classes were in a traditional format, while the third class was a guided session with content explanation in a VR environment.

The guided session aimed to reinforce the knowledge learned in traditional sessions prior in the week with a professor's explanation of the systems, functions, and parts in the VRE. The objective was a demonstration of the important concepts using the materials: VR device Oculus-Rift ${ }^{\circledR}$ and the VR software Organon 3D VR Anatomy ${ }^{\circledR}$

After the guided sessions, the students were assigned in teams of four members for performing a collaborative activity about the topic studied in that week.

The collaborative activities elaborated for the VR environment had the learning objectives:

- Identification of organs and important parts of the human system.

- Explanation of the process and functions of the human systems and their main organs.

- Explanation of diseases related to the human system and identification of affected organs.

\section{Stage 3. Knowledge reinforcement}

In the third stage, on session 9 and session 10 of the VRP, the students had an interaction with the professor focusing on their experience in the VRE while performing stage 2 (Virtual Reality Lab). An additional objective was to reinforce the knowledge content through direct interaction with the professor in review sessions. 


\section{Academic performance measurement}

\section{Final knowledge evaluation}

All the groups, the VRP groups (experimental) and the control groups, had a final knowledge evaluation where the students were tested for measuring their academic performance. This evaluation is normally applied for the subject with minor changes through the semesters.

The final knowledge evaluation consisted of 38 questions with 90 minutes for answering all the questions. The evaluation included topics from all the semester to evaluate the acquired knowledge in the subject.

The design of the exam covered the following sections: general questions about functions and characteristics of elements in human systems, identification of parts and organs within the human system, effects of environment and foreign elements in health, and short practical cases of human systems-related diseases.

\section{Academic performance analysis}

All grades were collected from the evaluation and transcribed in the software Microsoft Office 365 Excel@, where they were categorized by type of group.

A descriptive and inferential statistical analysis was made in Minitab®19. Outlier analysis was performed previous the statistical analysis, those points were removed from the data.

The descriptive statistics of the experimental and control groups were obtained. The distribution of grades for both types of groups was created in a histogram and fitting into a gamma distribution.

The inferential statistical analysis for experimental and control groups included a two-sample T-Test assuming unequal variances to determine the statistical difference between the results. Because there were two experimental groups (with same conditions) and two control groups a two-sample T-Test assuming unequal variances was performed too within the same type of groups.

\section{Results}

The outlier analysis showed one outlier in the VRP groups (experimental) and three in the control groups, in both situations the results corresponded to students with comparatively low academic performance. Referring to the specific students, they had a low performance during all the semester.

From the statistical analysis, relating to the academic performance in the final knowledge evaluation, the VRP groups obtained 77.24 as mean in comparison with 69.11 from the control groups. A two-sample t-test assuming unequal variances was performed for comparing the statistical difference between the means of the two types of groups, using a significance level of $\alpha=0.05$. There was a statistical difference with a p-value of 0.004 (Table 1). 
Table 1. Comparison of academic performance in final knowledge evaluation between VRP groups and Control groups, using two-sample t-test assuming unequal variances

\begin{tabular}{|l|l|l|l|l|l|l|l|l|}
\hline \multirow{2}{*}{ Method } & \multicolumn{9}{|l|}{ Descriptive Statistics } & \multicolumn{2}{l|}{ Test } \\
\cline { 5 - 9 } & \multicolumn{2}{l|}{ Null hypothesis } & $\mathrm{H}_{0}: \mu_{1}-\mu_{2}=0$ \\
\hline $\begin{array}{l}\mu_{1}: \text { population mean of } \\
\text { VRP }\end{array}$ & Sample & $\mathrm{N}$ & Mean & StDev & SE Mean & Alternative hypothesis & H: $\mu_{1}-\mu_{2} \neq 0$ \\
\hline $\begin{array}{l}\mu_{2} \text { : population mean of } \\
\text { Control }\end{array}$ & VRP & 63 & 77.24 & 14.1 & 1.8 & T-Value & DF & P-Value \\
\hline Difference: $\mu_{1}-\mu_{2}$ & Control & 57 & 69.11 & 16.2 & 2.1 & 2.92 & 111 & 0.004 \\
\hline
\end{tabular}

The academic performance was organized in different segments of grades aiming to identify trends of the groups. A descriptive statistical approach was used, fitting the data to a gamma distribution (Figure 4). From the results, it can be observed that most of the students in the VRP groups tend to higher grades, above 70, compared with the control groups. It is important to remark that in the institution where the VRP was implemented, 70 is the approval grade. That made the approval rate of the groups substantially different; at being $73.02 \%$ in the VRP groups compared with $43.86 \%$ from the control groups. In segments of the approval and non-approval sections, the percent of students from the VRP groups and controls groups was compared. Finding highest differences in segment 81-90 from the approval section, where $25.40 \%$ of the students from the VRP groups were against $12.29 \%$ from the control groups; while in the not-approval section, the largest difference was found in the segment 51-60 with $11.12 \%$ from VRP groups against $26.32 \%$ from control groups.

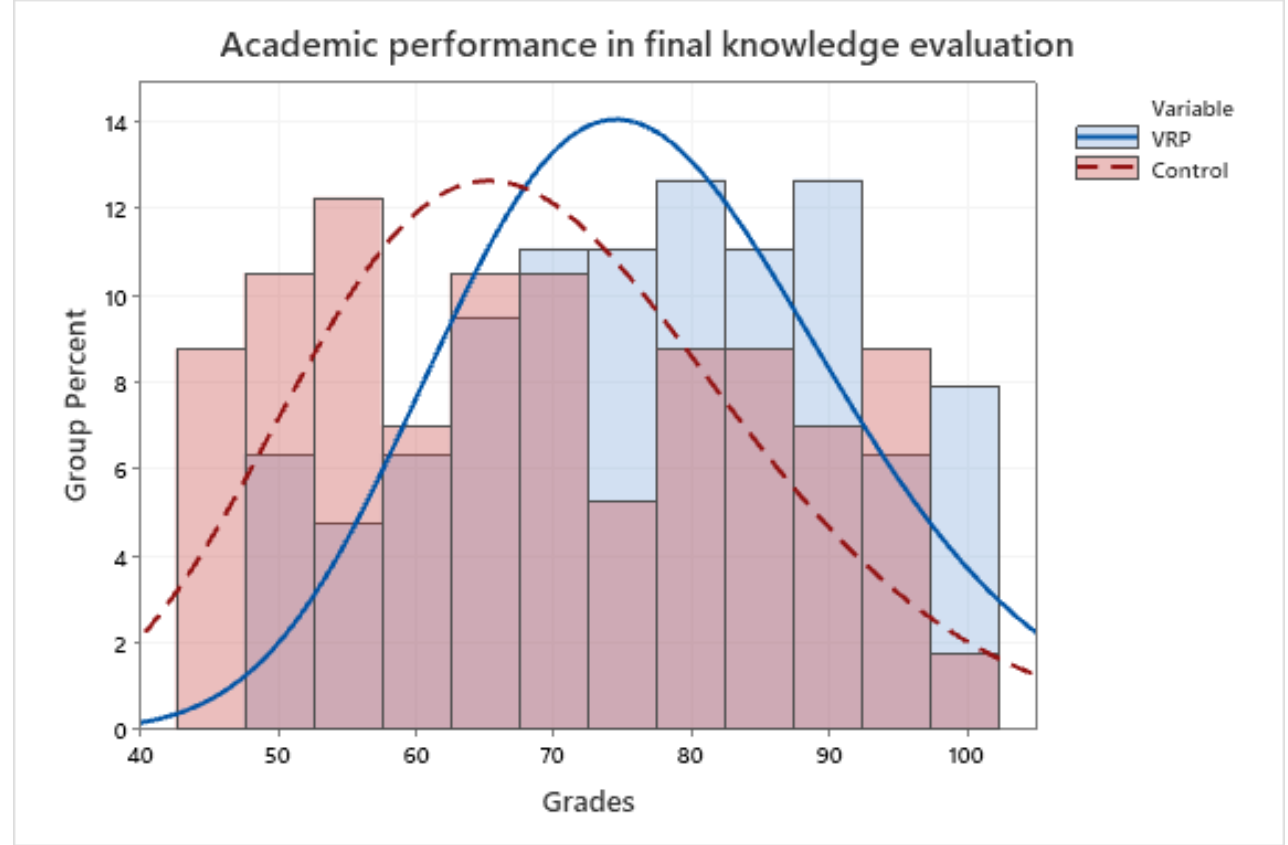

Figure 4. Academic performance's grades distribution, fitting a normal distribution, in final knowledge evaluation of VRP groups and control groups.

In the experimental design, two VRP groups and two control groups were considered. For knowing if there could be an effect of having two groups of each treatment, an inferential analysis for contrasting their means was conducted. A two-sample t-test assuming unequal variances was used for comparing the mean of each experimental group and the mean of each control group too. There was no statistical difference within both VRP groups, having 75.875 in group 1 and 78.645 for group 2 with a p-value of 0.438 (Table 2). Besides, there was no statistical difference in control group 1 with 70.469 and control group 2 with 67.360 with a p-value of 0.487 (Table 3). 
Table 2. Comparison of academic performance in final knowledge evaluation within VRP groups, using twosample t-test assuming unequal variances.

\begin{tabular}{|c|c|c|c|c|c|c|c|c|}
\hline \multirow{2}{*}{ Method } & \multirow{2}{*}{\multicolumn{5}{|c|}{ Descriptive Statistics }} & \multicolumn{3}{|l|}{ Test } \\
\hline & & & & & & \multirow{2}{*}{\multicolumn{2}{|c|}{$\begin{array}{l}\text { Null hypothesis } \\
\text { Alternative hypothesis }\end{array}$}} & \multirow{2}{*}{$\begin{array}{l}\mathrm{H}_{0}: \mu_{1}-\mu_{2}=0 \\
\mathrm{H}_{1}: \mu_{1}-\mu_{2} \neq 0\end{array}$} \\
\hline $\begin{array}{l}\mu_{1}: \text { population mean of } \\
\text { VRP } 1\end{array}$ & Sample & $\mathrm{N}$ & Mean & StDev & SE Mean & & & \\
\hline $\begin{array}{l}\mu_{2}: \text { population mean of } \\
\text { VRP } 2\end{array}$ & VRP 1 & 32 & 75.9 & 15.9 & 2.8 & $\begin{array}{l}\mathrm{T}- \\
\text { Value }\end{array}$ & DF & P-Value \\
\hline Difference: $\mu_{1}-\mu_{2}$ & VRP 2 & 31 & 78.6 & 12.1 & 2.2 & -0.78 & 57 & 0.438 \\
\hline
\end{tabular}

Table 3. Comparison of academic performance in final knowledge evaluation within Control groups, using twosample t-test assuming unequal variances.

\begin{tabular}{|c|c|c|c|c|c|c|c|c|}
\hline \multirow{2}{*}{ Method } & \multirow{2}{*}{\multicolumn{5}{|c|}{ Descriptive Statistics }} & \multicolumn{3}{|l|}{ Test } \\
\hline & & & & & & \multirow{2}{*}{\multicolumn{2}{|c|}{$\begin{array}{l}\text { Null hypothesis } \\
\text { Alternative hypothesis }\end{array}$}} & \multirow{2}{*}{$\begin{array}{l}\mathrm{H}_{0}: \mu_{1}-\mu_{2}=0 \\
\mathrm{H}_{1}: \mu_{1}-\mu_{2} \neq 0\end{array}$} \\
\hline $\begin{array}{l}\mu_{1}: \text { population mean of } \\
\text { Control } 1\end{array}$ & Sample & $\mathrm{N}$ & Mean & StDev & SE Mean & & & \\
\hline $\begin{array}{l}\mu_{2}: \text { population mean of } \\
\text { Control } 2\end{array}$ & Control 1 & 32 & 70.5 & 15.0 & 2.7 & T-Value & DF & P-Value \\
\hline Difference: $\mu_{1}-\mu_{2}$ & Control 2 & 25 & 67.4 & 17.7 & 3.5 & 0.70 & 47 & 0.487 \\
\hline
\end{tabular}

\section{Discussion}

The results indicate that using VR implementations with modified pedagogic strategies in long-time and collaborative interventions are effective for enhancing learning. It was observed a higher academic performance of the VRP groups than the control groups in the final knowledge evaluation, obtaining 77.24 as mean in comparison with 69.11 respectively. Also, the approval rate was considerably different; at being $73.02 \%$ in the VRP groups compared with $43.86 \%$ from the control groups. This supports the initial hypothesis that long-time and collaborative immersive VR implementations influence students' learning leading to higher academic performance in evaluations. The findings support results from other studies using VR for biology education, showing its effectiveness for enhancing learning because of its student-centered learning process (Lee \& Wong, 2014) and its positive impact improving conceptualization of complex biological process (Castéra et al., 2012).

There could be three factors that played a key role in enhancing learning in the VRP implementation: students' motivational level, collaborative interaction, and relation with the VR environment.

Motivation is crucial for learning and assuring the students' continuity. The use of VR increases the motivational level of students. According to Arslan et al. (2020), VR works at the motivational level, and the ability to animate events showing a positive impact on learning; especially, in fields like biology, anatomy, and physiology.

One important element of the VRP is the collaboration of students. In the implementation's second stage (VR $\mathrm{Lab}$ ), the students performed collaborative activities of human anatomy with a self-guided approach. It has been proved that VR can enhance collaborative creativity in different ways, including optimizing interactions with others and facilitating gamification guidance on the problem-solving process (Thornhill-Miller \& Dupont, 2016). 
Since early sessions in the implementation, the students showed optimist and little difficulty in managing the VR devices, this support previous research where students found VR environments easy to master without prior training and their practical approach is found amusing, attractive, and flexible for using audiovisual stimulation (Shudayfat et al.,2013). Besides, VR has been recognized as a technology that students are willing to use in educational environments (Kwun et al., 2019).

On the other hand, VR could be affecting the learning of students from a cognitive perspective. Students from the VRP improved their academic performance of topics early seen in the semester; considering the implementation was between week 5 and 15 and the final knowledge evaluation on week 17. It has been reported that VR improves cognitive processes, through three approaches: from perception and memory, stimulating the intensive development of a perception plan, and encouraging the development of specific cognitive motivation of a person. (Selivanov et al., 2020)

The VRP implementation focused on learning by academic performance, it is beyond the scope of this study to address the question of how long-time VR implementations have an impact on students' skills development, motivational level, and attitude toward the subject. Although the use of scientific skills is considered in the implementation it would be advisable to measure skills development for understanding the sources of changes in academic performance. The implementation considered the academic performance from a general result, it could be separated to identify in which subjects the impact was greater or lower. Measuring the attitude of students towards human biology would allow knowing changes in students' attitudes related to the implementation; there are designed instruments that could be applied, such as CLASS biology (Semsar et al., 2011)

\section{Conclusions}

This research aimed to identify the impact of long-time immersive VR implementations with a collaborative approach on learning in high school human biology course. Based on a quantitative analysis of academic performance, it can be concluded that this kind of VR implementation organized on progressive stages is effective in improving learning. The results indicate that the implementation increased the overall performance of the students and the approval rate of those groups.

This research illustrates that long-time VR implementations are good for improving learning by academic performance but also raise the questions of how VR affects the skills, motivation, and attitude of students in this kind of implementation. To better understand the implications of these results, future studies could address the effect on skills development measuring them on a pre-post test design. It is advisable to segment the topics in the final knowledge evaluation, allowing to re-evaluate the implementation in the topics with lower or no effect on the performance. Analyzing the attitude towards human biology from the students would give information to determine a change in students' attitude related to the implementation.

The findings from this research demonstrate how long-time and collaborative VR implementations can have a positive impact on academic performance and potentially on skills development, motivational level, and attitude towards the subject on the students. Focusing on analyzing academic performance could be a starting point for any implementation, continuing with measurements of the previously mentioned factors.

\section{Acknowledgments}

The author would like to acknowledge the financial support of Writing Lab, Institute for the Future of Education, Tecnologico de Monterrey, Mexico, in the production of this work.

The author thanks Rodrigo Ponce for his guidance and logistic support throughout the implementation; Erick Valdez for his assistance in the manuscript; the professors from the Natural Science Department for the 
activities' design and evaluations as well their assistance in the implementation; Cesar Camarillo for the activities' adaptation and collaboration in the implementation.

\section{References}

Arbona, C. B., García-Palacios, A., \& Baños, R. M. (2007). Realidad virtual y tratamientos psicológicos. Editorial Médica.

Arslan, R., Kofoğlu, M., \& Dargut, C. (2020). Development of Augmented Reality Application for Biology Education. Journal of Turkish Science Education (TUSED), 17(1), 62-72.

Bennett, J. A., \& Saunders, C. P. (2019). A virtual tour of the cell: Impact of virtual reality on student learning and engagement in the STEM classroom. Journal of microbiology \& biology education, 20(2).

Castéra, J., Sarapuu, T., \& Piksööt, J. (2012). Improving Student Conceptualisations through Manipulation in a Web-Based Learning Environment. eLearning \& Software for Education, 1, 449-454.

Escartín, E. R. (2000). La realidad virtual, una tecnología educativa a nuestro alcance. Pixel-Bit. Revista de Medios y Educación, 15, 5-21.

Fink, L. D. (2013). Creating Significant Learning Experiences: An Integrated Approach to Designing College Courses (2nd ed). John Wiley \& Sons, Incorporated.

Garcia, B. M., Jensen, M., \& Katona, G. (2019). A practical guide to developing virtual and augmented reality exercises for teaching structural biology. Biochemistry \& Molecular Biology Education, 47(1), 16-24.

Human Anatomy VR® (2020). Playstation®. Retrieved on December 10, 2020 from https://store.playstation.com/es-mx/product/UP5269-CUSA16456_00-VIRTUALMEDICINEX

Kwun, O., Alijani, G. S., \& Khaleel, M. A. (2019). Adoption of Virtual Reality in Learning among Hbcu Students. Global Journal of Business Pedagogy (GJBP), 3(1), 116-124.

Lee, E. A.-L., \& Wong, K. W. (2014). Learning with desktop virtual reality: Low spatial ability learners are more positively affected. Computers \& Education, 79, 49-58.

Martínez, F. P. (2011). Presente y Futuro de la Tecnología de la Realidad Virtual. Creatividad y sociedad.

Mason, H. D. (2017). Sense of meaning and academic performance: A brief report. Journal of Psychology in Africa, 27(3), 282-285.

Oculus Go® - Standalone Virtual Reality Headset (2020). Oculus by Facebook®. Retrieved on December 10, 2020 from https://www.oculus.com/

Oculus Rift $\AA^{-}$Standalone Virtual Reality Headset (2020). Oculus by Facebook®. Retrieved on December 10, 2020 from https://www.oculus.com/rift-s/

Organon 3D VR Anatomy® (2020). Medis Media®. Retrieved on December 10, 2020 from https://www.amazon.com/-/es/dp/B07WGR2VBP

Selivanov, V. V., Selivanova, L. N., \& Babieva, N. S. (2020). Cognitive Processes and Personality Traits in Virtual Reality Educational and Training. Psychology in Russia. State of the Art, 13(2), 16-28.

Semsar, K., Knight, J., Birol, G. \& Smith, M. (2011). The Colorado Learning Attitudes about Science Survey (CLASS) for Use in Biology. Life Sciences Education, (10), 268-278.

Shim, K. C., Park, J. S., Kim, H. S., Kim, J. H., Park, Y. C., \& Ryu, H. I. (2003). Application of virtual reality technology in biology education. Journal of Biological Education, 37(2), 71-74.

Shudayfat, E. A., Moldoveanu, A., Moldoveanu, F., \& Grădinaru, A. (2013). Virtual Reality-Based Biology Learning Module. ELearning \& Software for Education, 2, 621-626.

Solak, E., \& Erdem, G. (2015). A Content Analysis of Virtual Reality Studies in Foreign Language Education. Participatory Educational Research, 21-26.

Steuer, J. (1992). Defining virtual reality: Dimensions determining telepresence. Journal of communication, 42(4), 73-93. 
Thornhill-Miller, B., \& Dupont, J.-M. (2016). Virtual Reality and the Enhancement of Creativity and Innovation: Under Recognized Potential Among Converging Technologies? Journal of Cognitive Education \& Psychology, 15(1), 102-121.

Vicerrectoria Académica ITESM (2014), Planes de studio PrepaTec Multicultural. Retrieves on December 10, 2020 from https://samp.itesm.mx/Materias/VistaPreliminarMateria?clave=PC2000\&lang=EN\#

Youngblut, Ch. (1998). Educational Uses of Virtual Reality Technology. Institute for Defense Analysis. 